\title{
Early Outcomes of Obese Patients Undergoing Total Hip Arthroplasty: Comparison of Anterior to Posterior Approach
}

\author{
Eric N. Windsor, William D. Zelenty, Blake M. Bodendorfer ${ }^{*}$, Richard Verstraete, Mark W. Zawadsky ${ }^{\mathrm{i}}$ \\ Department of Orthopaedic Surgery, MedStar Georgetown University Hospital, USA
}

Copyright $\bigcirc 2019$ by authors, all rights reserved. Authors agree that this article remains permanently open access under the terms of the Creative Commons Attribution License 4.0 International License

\begin{abstract}
BACKGROUND: There has been an increase in total hip arthroplasty in obese patients and surgical complication rates are known to be higher in this population. This retrospective study evaluated complication rates for obese patients undergoing total hip replacement compared to non-obese patients. Additionally, we compared complication rates for direct anterior approach (ATHA) versus posterior approach (PTHA). METHODS: This study was an IRB approved retrospective review of 210 patients undergoing ATHA and 201 patients undergoing PTHA during the same time period by 2 total joint surgeons. Non-obese patients were compared to obese patients using body mass index (BMI) classification. Minor and major complications were reviewed as well as length of stay, disposition, and other short-term outcome measures. RESULTS: The non-obese cohort $(\mathrm{BMI}<30)$ had lower complication rates $(2.8 \%$ major, $4.4 \%$ minor) compared to the obese cohort $(8.7 \%$ major, $9.9 \%$ minor). Major complications by obese class were: Class I $8.6 \%$, Class II $7.1 \%$ and Class III $11.5 \%$. The non-obese ATHA cohort had lower complication rates ( $0.8 \%$ major, $5.0 \%$ minor) compared to the PTHA cohort (5.0\% major, $6.7 \%$ minor). Obese patients had $6.3 \%$ major and $9.9 \%$ minor complication rates in ATHA, compared to $11.1 \%$ major and $10.0 \%$ minor complication rates in PTHA. Lastly, the evaluation of short-term outcomes showed more favorable results for ATHA for both obese and non-obese patients. CONCLUSION: Obesity was associated with an increased risk of complications and less favorable short-term outcomes following THA. Direct anterior THA was associated with lower complication rates and more favorable short-term outcomes.
\end{abstract}

Keywords Total Hip Arthroplasty, Obesity, Anterior Approach, Posterior Approach, Perioperative Complications

\section{Introduction}

The obesity crisis combined with the aging population in the United States has caused a significant increase in the prevalence of hip arthritis in recent years and has led to an increased incidence of total hip arthroplasty. Surgical complication rates are known to be higher in the obese patient population and guidelines are being considered as to which patients have a risk profile that allows them to be acceptable candidates for surgical intervention $[1,2]$. The risk of various peri-operative complications in obese patients includes infection, wound healing issues, peri-prosthetic mal-alignment or fracture, medical complications, and thromboembolic events [2-6]. There are a limited number of studies in the literature that provide specific data on the actual complication rates for total hip replacement surgery in obese patients [7-9], and fewer that specifically evaluate the different complication rates associated with the surgical approach used in obese patients [10-13].

Total hip arthroplasty through a direct anterior approach has gained recent attention and popularity with both patients and surgeons due to its potential for faster recovery, increased stability, and decreased pain when compared with other surgical approaches [14-16]. However, what is often underestimated is the high degree of difficulty associated with exposure in the obese patient, as well as the direct correlation between surgical approach and complication rates in this patient population [14]. These issues are also problematic in the more common posterior approach for total hip arthroplasty.

We have previously reported on the complication rates and early outcomes measures using the direct anterior approach, and found that there was a higher complication rate and slower recovery rate for obese patients compared to non-obese patients [17]. Our findings also suggested that the direct anterior approach compared favorably to the published data in the literature for the posterior approach in 
the obese population. Therefore, the purpose of this study was to evaluate complication rates and short-term outcomes of obese and non-obese patients undergoing THA with a direct anterior approach in comparison to a similar consecutive group of patients undergoing THA with a posterior approach, during the same time period at the same institution using similar patient care protocols. This study also provides actual complication rates associated with THA broken down by approach and obese classification.

\section{Methods}

This study is an Institutional Review Board approved retrospective review of 210 consecutive patients undergoing a direct anterior approach total hip arthroplasty along with 201 consecutive patients undergoing a posterior approach total hip arthroplasty by a different surgeon. There were 161 patients in total with a BMI greater than 30 , with 80 of these obese patients in the anterior approach group, and 81 in the posterior approach group. The cases were completed during the same time period by experienced, fellowship trained, high volume total joint surgeons in a demographically similar group of patients. This was a retrospective review of hospital and clinical medical records. Intraoperative and early (first 3 months) postoperative complications were evaluated, as well as various outcome data. Normal and pre-obese patients were compared to obese patients using WHO categories for BMI classification. The WHO Classification of obesity is as follows: normal: $<25 \mathrm{~kg} / \mathrm{m}^{2}$, pre-obese: $25-29.9 \mathrm{~kg} / \mathrm{m}^{2}$, class I: $30-34.9 \mathrm{~kg} / \mathrm{m}^{2}$, class II: $35-39.9 \mathrm{~kg} / \mathrm{m}^{2}$, class III: > $40.0 \mathrm{~kg} / \mathrm{m}^{2}$. Minor and major complications were reviewed, as well as surgical time, length of stay, disposition, and other short-term outcome measures.

All of the cases used similar implants, which included a Pinnacle uncemented acetabular component, a highly cross-linked polyethylene liner, and either a cobalt chrome or Biolox Delta ceramic femoral head of $32 \mathrm{~mm}$ or $36 \mathrm{~mm}$ in size. The femoral implants were a Trilock BPS uncemented stem for the anterior cases, and a Summit uncemented stem for the posterior cases (all Depuy, Warsaw, IN).

The direct anterior approach utilized a modified SmithPeterson interval as described by Berend et al [16]. The approach is between the tensor fascia lata muscle and the sartorius, with deep dissection extending lateral to the rectus femoris. A standard operating table was used along with a femoral elevator allowing for proper femoral exposure (Omni-Tract Surgical, St. Paul, MN), as well as dual-offset broach handles to help with femoral canal preparation. Routine use of fluoroscopy was not used.

The mini-posterior approach was through a postero-lateral incision as described by Sculco et al [18]. The patient is placed in a lateral decubitus position on a standard operating table, the fibers of the Gluteus Maximus are split longitudinally, and the posterior rotators are detached along with the posterior capsule and then closed through drill holes in the greater trochanter.

Unless contraindicated, patients for both the anterior and posterior cases were treated with a standardized pain protocol in combination with regional anesthesia at the discretion of the anesthesia team. A local tissue pain injection was administered as well as intravenous tranexamic acid. The same closure technique was utilized for all patients, which included a \#1 or \#2 barbed suture to close the deep fascia and subcutaneous tissue, and a running 0 barbed suture for the skin. Dermabond (Ethicon, Sommerville, NJ) was applied topically and an occlusive foam dressing was applied (Mepilex, Gothenberg, Sweden).

Physical therapy followed a standardized protocol for all patients and began on postoperative day 0 with mobilization out of bed, followed by twice daily treatments thereafter, progressing from a walker to a cane to no assistive devices as tolerated by the patient. Patients were discharged when they were able to safely mobilize for daily activities and cleared by physical therapy, their pain was controlled on oral medications, and they were medically stable. Standard post-discharge follow-up was at two and six weeks. All patients were placed on aspirin $325 \mathrm{mg}$ twice daily for DVT prophylaxis or Warfarin if indicated after risk stratification.

Electronic and paper medical records were reviewed to determine surgical time, length of stay, discharge disposition, major and minor complications, and short-term outcome measures. All patient charts were available and no patients were lost to follow up in the immediate post-operative period. Major complications were defined as venous thromboembolism (VTE), wound infection or periprosthetic fracture requiring return to the operating room, hip dislocation, significant medical complications, or any adverse event requiring return to the operating room in the 3 months following surgery. Minor complications included wound drainage or delayed healing requiring dressing changes or oral antibiotics without a return to the operating room, or other minor medical complications.

\section{Statistics}

Statistical interpretation of the data was carried out by comparing the cohorts of non-obese patients versus obese patients (BMI > 30), and then also between the anterior approach patients versus posterior approach patients according to obesity classification level. Continuous variables are presented using means and standard deviations, and comparison between groups means is conducted using ANOVA. If continuous measures do not have a normal distribution, then data is presented using median, $25^{\text {th }}$ and $75^{\text {th }}$ percentile, and non-parametric Kruskal Wallis test is used for comparison of groups. 
Categorical data is presented using frequency and percentages, and comparison between groups is conducted using chi square test or fisher's exact test in case of small cell sizes.

\section{Results}

Significant findings showed that in a comparison of the non-obese group $(\mathrm{BMI}<30)$ to the obese group $(\mathrm{BMI}>30)$ for the overall cohort of 411 patients, major complication rates were $2.8 \%$ versus $8.7 \%$ ( $\mathrm{p}$ value $=0.0155$ ), and minor complication rates were $4.4 \%$ versus $9.9 \%$ ( $\mathrm{p}$ value $=$ 0.0446), respectfully. Major complications by obese category were Class I: $8.6 \%$, Class II: $7.1 \%$, and Class III: $11.5 \%$. Minor complications by obese category were Class I: $7.5 \%$, Class II: $2.4 \%$, and Class III: $30.8 \%$. Patient demographics were well matched between the groups (Table 1).

Table 1. Demographic Data $(n=411)$

\begin{tabular}{|c|c|c|c|c|}
\hline Variable & Total $n=411$ & Approach Anterior $\mathrm{n}=210$ & Approach Posterior $n=201$ & $\mathrm{p}$ \\
\hline Procedure n $(\%)$ & & & & 0.61 \\
\hline L THA & $190(46.2)$ & $94(44.8)$ & $96(47.8)$ & \\
\hline R THA & $221(53.8)$ & $116(55.2)$ & $105(52.2)$ & \\
\hline BMI mean(sd) & $29.27(6.5)$ & $29(5.93)$ & $29.55(7.05)$ & 0.396 \\
\hline Age at surgery mean(sd) & $61(11.1)$ & $61.06(10.95)$ & $60.95(11.27)$ & 0.915 \\
\hline Obesity Class n (\%) & & & & 0.32 \\
\hline Non-Obese & $116(28.2)$ & $61(29)$ & $55(27.4)$ & \\
\hline Pre-Obese & $134(32.6)$ & $69(32.9)$ & $65(32.3)$ & \\
\hline Class I & $93(22.6)$ & $49(23.3)$ & 44 (21.9) & \\
\hline Class II & $42(10.2)$ & $23(11)$ & $19(9.5)$ & \\
\hline Class III & $26(6.3)$ & $8(3.8)$ & $18(9)$ & \\
\hline
\end{tabular}

Table 2. Outcomes by Approach - Non/Pre-Obese

\begin{tabular}{|c|c|c|c|c|}
\hline Variable & Total & Anterior $\mathrm{n}=130$ & Posterior $n=120$ & $\mathrm{p}$ \\
\hline OR Time median(IQR) $(n=250)$ & $70(62-80)$ & $74(65-85)$ & $66(60-76)$ & $<0.001$ \\
\hline ASA median $(\mathrm{IQR})(\mathrm{n}=250)$ & $2.2(2-2)$ & $2(2-2)$ & $2(2-3)$ & 0.248 \\
\hline VAS Pre-Surgery median(IQR) $(\mathrm{n}=249)$ & $7(5-8)$ & $7(6-8)$ & $6(5-8)$ & 0.086 \\
\hline Minor Complications $n(\&)(n=250)$ & & & & 0.17 \\
\hline No & $239(95.6)$ & $127(97.9)$ & $112(93.3)$ & \\
\hline Yes & $11(4.4)$ & $3(2.3)$ & $8(6.7)$ & \\
\hline Major Complications $n(\%)(n=250)$ & & & & 0.10 \\
\hline No & $243(97.2)$ & $129(99.2)$ & $114(95)$ & \\
\hline Yes & $7(2.8)$ & $1(0.8)$ & $6(5)$ & \\
\hline Narcotic Use 2 weeks n $(\%)(n=250)$ & & & & $<0.001$ \\
\hline No & $140(56)$ & $97(74.6)$ & $43(35.8)$ & \\
\hline Yes & $110(44)$ & $33(25.4)$ & $77(64.2)$ & \\
\hline Narcotic Use 6 weeks n $(\%)(n=250)$ & & & & $<0.001$ \\
\hline No & $222(88.8)$ & $126(96.9)$ & $96(80)$ & \\
\hline Yes & $28(11.2)$ & $4(3.1)$ & $24(20)$ & \\
\hline Assistive Device Use 2 weeks n (\%) $(\mathrm{n}=250)$ & & & & $<0.001$ \\
\hline No & $66(26.4)$ & $47(36.2)$ & $19(15.8)$ & \\
\hline Yes & $184(73.6)$ & $83(63.8)$ & $101(84.2)$ & \\
\hline Assistive Device Use 6 weeks n (\%) $(\mathrm{n}=250)$ & & & & 0.88 \\
\hline No & $202(80.8)$ & $106(81.5)$ & $96(80)$ & \\
\hline Yes & $48(19.2)$ & $24(18.5)$ & $24(20)$ & \\
\hline
\end{tabular}


Table 3. Outcomes by Approach - Obese

\begin{tabular}{|c|c|c|c|c|}
\hline Variable & Total & Anterior $\mathrm{n}=80$ & Posterior $\mathrm{n}=81$ & $\mathrm{p}$ \\
\hline OR Time median(IQR) $(\mathrm{n}=160)$ & $78(68-93)$ & $88(73-100)$ & $72(66-84)$ & $<0.001$ \\
\hline ASA median $(\mathrm{IQR})(\mathrm{n}=161)$ & $2(2-3)$ & $2(2-3)$ & $2(2-3)$ & 0.935 \\
\hline VAS Pre-Surgery median(IQR) $(n=161)$ & $8(6-9)$ & $8(7-9)$ & $8(6-9)$ & 0.264 \\
\hline Minor Complications $\mathrm{n}(\%)(\mathrm{n}=161)$ & & & & 1.00 \\
\hline No & $145(90.1)$ & $72(90)$ & $73(90.1)$ & \\
\hline Yes & $16(9.9)$ & $8(10)$ & $8(9.9)$ & \\
\hline Major Complications n $(\%)(n=161)$ & & & & 0.42 \\
\hline No & $147(91.3)$ & $75(93.8)$ & $72(88.9)$ & \\
\hline Yes & $14(8.7)$ & $5(6.2)$ & $9(11.1)$ & \\
\hline Narcotic Use 2 weeks n (\%) $(n=161)$ & & & & $<0.001$ \\
\hline No & $66(41)$ & $45(56.2)$ & $21(25.9)$ & \\
\hline Yes & $95(59)$ & $35(43.8)$ & $60(74.1)$ & \\
\hline Narcotic Use 6 weeks n (\%) $(n=161)$ & & & & 0.004 \\
\hline No & $134(83.2)$ & $74(92.5)$ & $60(74.1)$ & \\
\hline Yes & $27(16.8)$ & $6(7.5)$ & $21(25.9)$ & \\
\hline Assistive Device Use 2 weeks n (\%) $(\mathrm{n}=161)$ & & & & 0.47 \\
\hline No & $22(13.7)$ & $13(16.2)$ & $9(11.1)$ & \\
\hline Yes & $139(86.3)$ & $67(83.8)$ & $72(88.9)$ & \\
\hline Assistive Device Use 6 weeks n (p) $(n=161)$ & & & & 0.42 \\
\hline No & $113(70.2)$ & $59(73.8)$ & $54(66.7)$ & \\
\hline Yes & $48(29.8)$ & $21(26.2)$ & $27(33.3)$ & \\
\hline
\end{tabular}

Table 4. Major Complications by Approach

\begin{tabular}{|c|c|c|}
\hline & Anterior Approach & Posterior Approach \\
\hline \multirow[t]{2}{*}{ Non-obese } & 1 Infection (I\&D Retained Implants) & $\begin{array}{l}\text { 1 Infection (I\&D Retained Implants) } \\
1 \text { Infection (Two Stage Explant) } \\
1 \text { Intra-Op Fracture } \\
3 \text { Medical (PE, Cardiac, Seizures) }\end{array}$ \\
\hline & 1 Total & 6 Total \\
\hline \multirow[t]{2}{*}{ Obese } & $\begin{array}{l}2 \text { Infection (Two Stage Explant) } \\
1 \text { Intra-Op Fracture } \\
2 \text { Stem Revision (Subsidence) }\end{array}$ & $\begin{array}{l}1 \text { Infection (I\&D Retained Implants) } \\
1 \text { Intra-Op Fracture } \\
3 \text { Dislocation } \\
4 \text { Medical (PR, Renal Failure, Mortality) }\end{array}$ \\
\hline & 5 Total & 9 Total \\
\hline
\end{tabular}

When comparing the anterior approach to the posterior approach, there was a trend favoring the anterior approach, although statistical significance was not reached (Tables 2, $3,4)$. In the non-obese patients (BMI < 30), major complication rates for the anterior approach were $0.8 \%$ compared to $5.0 \%$ for the posterior approach ( $\mathrm{p}$ value $=$ $0.10)$. Minor complication rates were $2.3 \%$ in the anterior group compared to $6.7 \%$ for the posterior group $(\mathrm{p}$ value $=$ $0.17)$. Obese patients $(\mathrm{BMI}>30)$ had major complication rates of $6.3 \%$ in the anterior group compared to $11.1 \%$ in the posterior group ( $\mathrm{p}$ value $=0.42$ ), and minor complication rates were similar at $9.9 \%$ for the anterior group and $10.0 \%$ for the posterior group ( $\mathrm{p}$ value $=1.0$ ).

The evaluation of short term outcomes showed more favorable results for the anterior approach group compared to the posterior approach group in non-obese patients. Hospital length of stay was shorter for the anterior group at 2.32 days versus 2.63 days for the posterior group ( $\mathrm{p}$ value $=0.034$ ) (Figure 1), and discharge to inpatient rehabilitation was lower for the anterior group at $7.7 \%$ versus $20.8 \%$ (p value $<0.005$ ) (Figure 2). Narcotic use at 2 weeks was lower for the anterior group $(25.4 \%$ versus $64.2 \%$; p value $<0.001$ ) (Figure 3 ), as well as at 6 weeks $(3.1 \%$ versus $20.0 \%$; $p$ value $<0.001)$. VAS pain scores at 2 weeks were lower for the anterior group at 2.05 versus 2.89 ( $\mathrm{p}$ value $<0.047$ ), but similar at 6 weeks (Figure 4). The use of assistive devices at 2 weeks was $63.8 \%$ for the anterior group versus $84.2 \%$ for the posterior group ( $p$ value $<0.001$ ). 


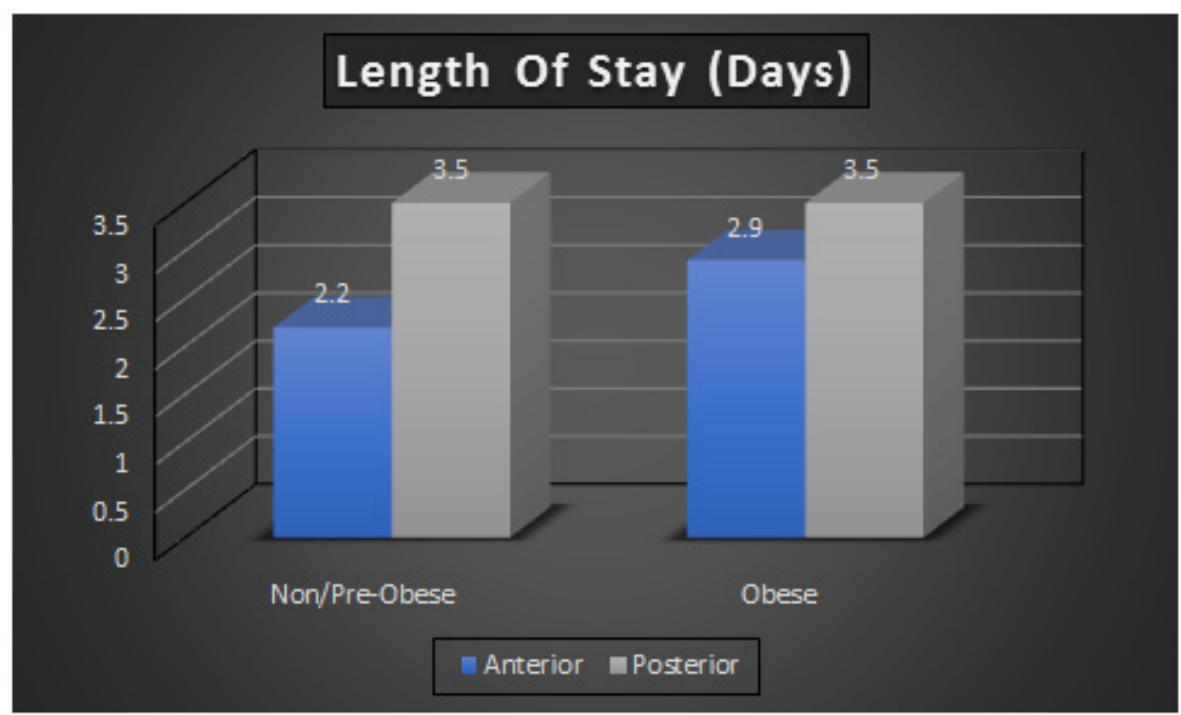

Figure 1. Length of Stay Comparison

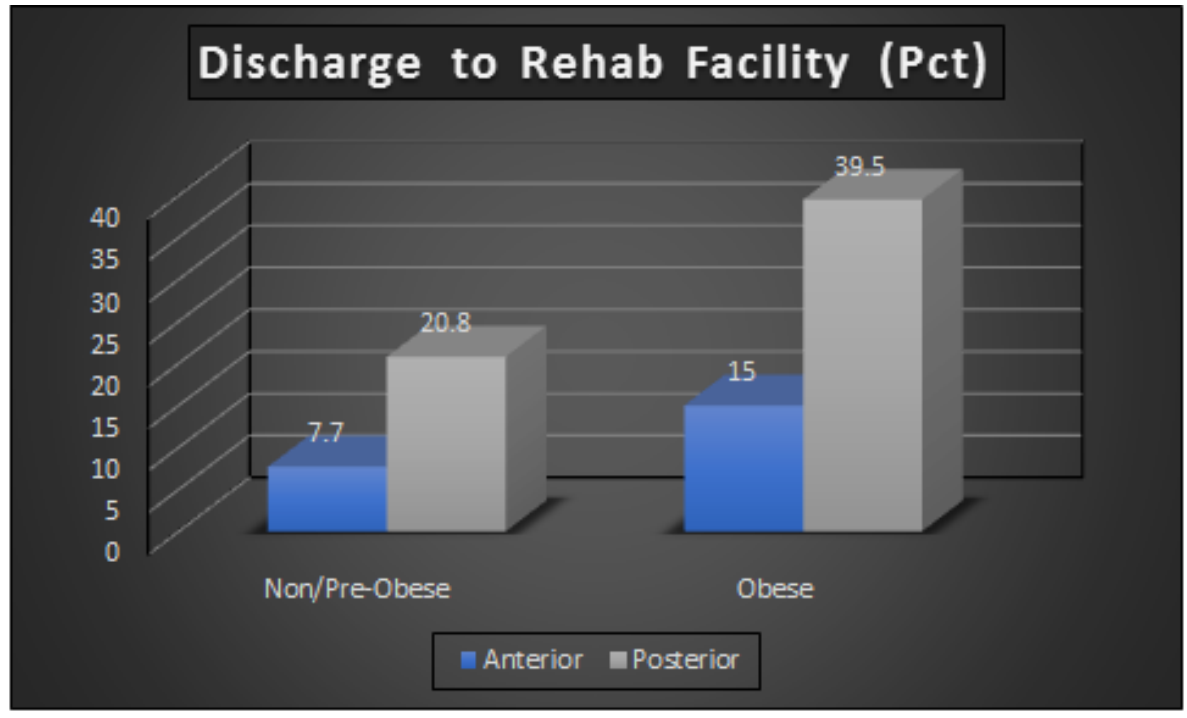

Figure 2. Discharge Disposition Comparison

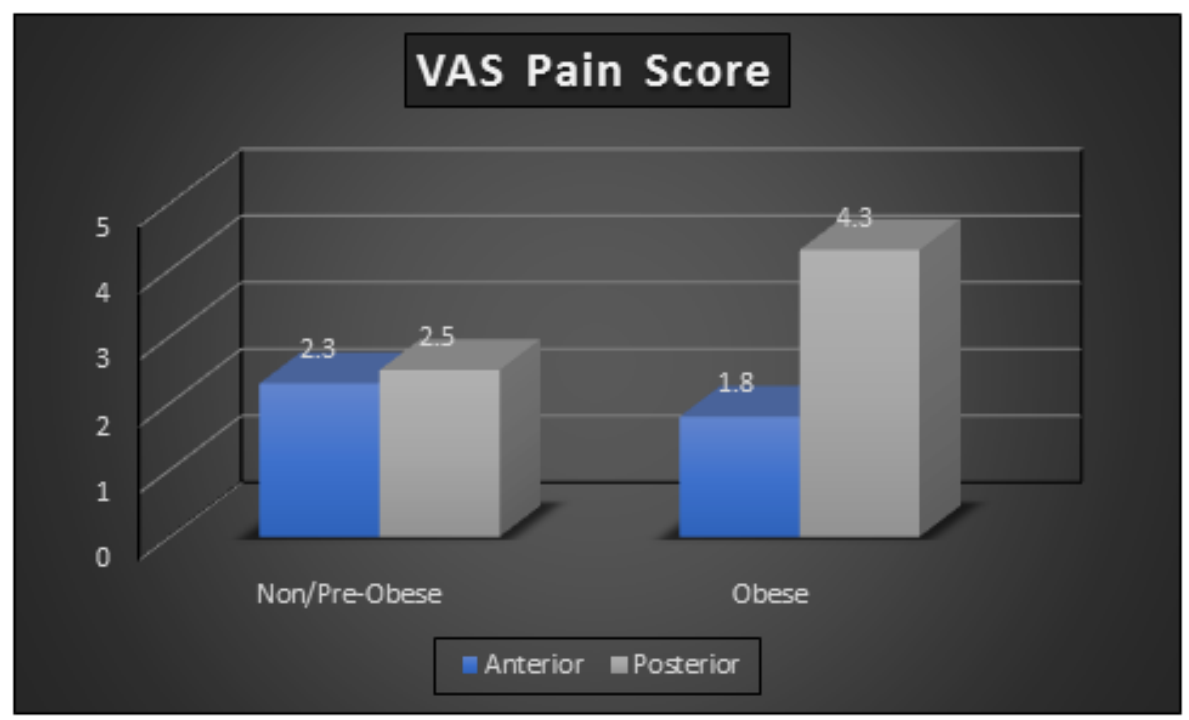

Figure 3. VAS Pain Score Comparison 


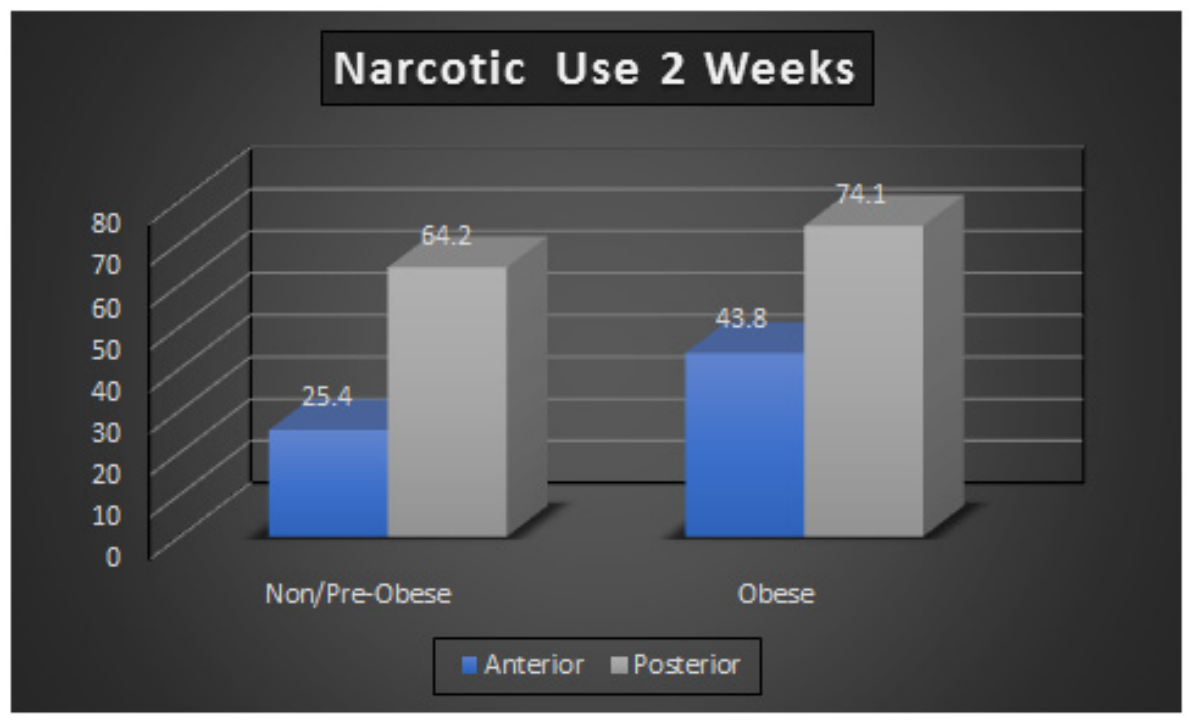

Figure 4. Narcotic Use at 2 Weeks Comparison

In the obese group, patients with a BMI $>30$, the anterior group also had favorable findings. Discharge to inpatient rehabilitation was lower for the anterior group $(15.0 \%$ versus $39.5 \%$; $p$ value $<0.001$ ). Hospital length of stay was shorter for the anterior group at 2.63 compared to 2.96, but did not reach statistical significance $(\mathrm{p}$ value $=0.13$ ). VAS pain scores at 2 weeks were lower for the anterior group at 2.79 versus 4.24 ( $\mathrm{p}$ value $<0.001$ ), but similar at 6 weeks (1.93 anterior versus 1.74 posterior; $p$ value $=0.54$ )

\section{Discussion}

This study evaluated the effect of obesity on the complication rates and short-term outcomes in total hip arthroplasty patients, along with a comparison between the anterior and posterior approaches used for the surgery. Our results show that complication rates increase significantly for obese patients when compared to non-obese patients regardless of the approach used. We also found that the anterior approach had a trend toward more favorable outcomes and lower complication rates in both obese and non-obese patients when compared to the posterior approach, with both procedures being performed at our institution using similar peri-operative protocols among a well-matched cohort of patients. The lower use of inpatient rehabilitation placement along with better patient comfort and mobility amongst the anterior group may infer a cost benefit, which can be useful with the current focus on cost savings in today's medical environment.

Another important finding was that for patients with Class I obesity (BMI $=30.0$ to 34.9), the major complication rate increased to $8.6 \%$ compared to $2.8 \%$ in non-obese patients. In class III patients with a BMI $>40$, the complication rate was $11.5 \%$ for major complications and $30.8 \%$ for minor complications, although the group was a small cohort of only 26 patients (8 ATHA and 18
PTHA). The numbers available in the obese categories were relatively small, which may have led to the lack of statistical significance in some of the findings since there was a consistent trend across several measures.

With the incidence of obesity rapidly increasing, along with the rising need for THA in this patient population, orthopaedic surgeons and healthcare systems will need to be prepared to manage these patients and their complications. Surgeons will require as many assessment and decision-making tools as possible to appropriately select patients and mitigate complications. The findings in this study add to the literature by means of providing actual complication rates and short-term outcomes data for both non-obese and obese patients, as well as comparison data about the impact of surgical approach used for the surgery. This should help to inform both the surgeon and patient about the risks associated with this procedure.

Obese patients are a challenging cohort of patients due to their large body habitus [19-22]. One advantage of the anterior approach in comparison to the posterior approach is that many obese patients have a relatively small amount of subcutaneous adipose tissue along the anterior aspect of the thigh, which allows for a relatively routine operative procedure [14, 23-24]. This may account for some of the advantages that we found in this study. We have also found that moving the proximal aspect of the incision more lateral to avoid the inguinal crease, and taping the pannus towards the contralateral hip, can significantly help the exposure.

This study demonstrated complication rates amongst obese patients in accordance with existing literature. Michalka et al. [7] showed a complication rate of $14.0 \%$ and $12.3 \%$ for major and minor events, respectively, in obese patients within 6 weeks using a posterior approach. In a prospective matched cohort study using an anterolateral approach, Chee et al. [8] reported a 9\% major complication rate and a $13 \%$ minor wound complication rate for their obese patients, who had an average BMI of 
37.9. Our data showed a major complication rate of $8.7 \%$ and a minor complication rate of $9.9 \%$ for obese patients.

A study by Dowsey and Choong [9] of obese patients using a posterior approach reported a $4.4 \%$ acute infection rate in comparison to our rate of $1.2 \%$ in the obese posterior group. A study by Purcell et al. [12] showed that obesity was a more significant risk factor than the approach used in terms of infection and wound complications when comparing the anterior and posterior approaches. They found infection rates of $2.35 \%$ for the anterior approach and $2.7 \%$ for the posterior approach, which are similar to the infection rates in our study (2.5\% for the anterior group and $1.2 \%$ for the posterior group). Ponzio et al [13] found a lower dislocation rate for the anterior approach at $0.4 \%$ compared to $1.5 \%$ for the posterior approach. This was similar to our rates of $0 \%$ for the anterior and $1.5 \%$ for the posterior approach.

Oosting et al. [25] examined recovery after THA as a function of obesity and showed that obese patients required longer stays and had a prolonged recovery period to normal function. Our obese cohorts did have longer average stays than the non-obese cohorts ( 2.6 days versus 2.3 days in the anterior group, and 2.96 days versus 2.63 days in the posterior group). Though it was beyond the scope of our study, obesity is similarly associated with higher rates of late complications and failure. Hanna et al. [26] demonstrated that Class III obesity is associated with higher rates of failure, lower functional scores, and greater need for revision procedures when compared to Class II obese patients ( $27 \%$ versus $12 \%$, respectively). Our study showed a major complication rate of $11.5 \%$ and minor complication rate of $30.8 \%$ in the class III obese patients during the early peri-operative period.

The strength of this study is that the patients were well matched in terms of demographics, and were treated with similar peri-operative protocols at one institution during the same time period, which helps to reduce selection bias from the study. However, there were also several limitations of this study. First, it was a retrospective cohort analysis, which could be subject to bias even though demographics were well matched and the patient pool was similar in scope. Likewise, while we do use a standard pain regimen postoperatively, preoperative pain management was not standardized and postoperative changes to pain regimens after discharge could have varied as well. This study only has short term follow-up, which may cause us to underestimate the incidence of certain complications, but our intent was to examine the early peri-operative time period. We did not examine standard patient reported outcomes measures beyond VAS score, which could be valuable for future study. Lastly, we did not perform $a$ priori power analysis; while there was a trend towards more complications in the PTHA group, this did not achieve significance as the study was likely underpowered due to the relatively small numbers in the obese cohorts.

\section{Conclusions}

This study demonstrates that the risk of complication in the peri-operative period after total hip replacement increases significantly in the obese population when compared to non-obese patients. Short term outcomes measures were less favorable in the obese group. In addition, patients undergoing a direct anterior approach had a lower rate of complications following surgery in comparison to the posterior approach for both non-obese as well as obese patients. The anterior approach also showed more favorable early outcomes when compared to the posterior approach.

\section{REFERENCES}

[1] Maradit Kremers H, Kremers HM, Visscher SL, Kremers WK, Naessens JM, Lewallen DG. Obesity Increases Length of Stay and Direct Medical Costs in Total Hip Arthroplasty. Clin Orthop Relat Res. 472(4):1232-1239, 2013.

[2] Pugely AJ, Callaghan JJ, Martin CT, Cram P, Gao Y. Incidence of and risk factors for 30-day readmission following elective primary total joint arthroplasty: analysis from the ACS-NSQIP. J Arthroplasty. 28(9):1499-1504, 2013.

[3] Goodnough LH, Finlay AK, Huddleston JI 3rd, Goodman SB, Maloney WJ, Amanatullah DF. Obesity Is Independently Associated With Early Aseptic Loosening in Primary Total Hip Arthroplasty. J Arthroplasty. 33(3):882-886, 2018.

[4] Gulotta LV, Padgett DE, Sculco TP, Urban M, Lyman S, Nestor BJ. Fast Track THR: One Hospital's Experience with a 2-Day Length of Stay Protocol for Total Hip Replacement. HSS J. 7(3): 223-228, 2011.

[5] Smith JO, Frampton CMA, Hooper GJ, Young SW. The Impact of Patient and Surgical Factors on the Rate of Postoperative Infection after Total Hip Arthroplasty-A New Zealand Joint Registry Study. J Arthroplasty. January 2018.

[6] Triantafyllopoulos GK, Soranoglou VG, Memtsoudis SG, Sculco TP, Poultsides LA. Rate and Risk Factors for Periprosthetic Joint Infection among 36,494 Primary Total Hip Arthroplasties. J Arthroplasty. November 2017.

[7] Michalka PKR, Khan RJK, Scaddan MC, Haebich S, Chirodian N, Wimhurst JA. The Influence of Obesity on Early Outcomes in Primary Hip Arthroplasty. J Arthroplasty. 27(3):391-396, 2012.

[8] Chee Y, Teoh K, Sabnis B, et al. Total hip replacement in morbidly obese patients with osteoarthritis. J Bone Joint Surgery (Br) 92-B: 1066, 2010.

[9] Dowsey M, Choong P. Obesity is a major risk factor for prosthetic infection after primary hip arthroplasty. Clin Orthop Relat Res, 466:153, 2008. 
[10] Joseph NM, Roberts J, Mulligan MT. Financial impact of total hip arthroplasty: a comparison of anterior versus posterior surgical approaches. Arthroplasty Today, 3(1):39-43, 2008.

[11] Hanly RJ, Marvi SK, Whitehouse SL, Crawford RW. Morbid Obesity in Total Hip Arthroplasty: Redefining Outcomes for Operative Time, Length of Stay, and Readmission. J Arthroplasty. 31(9):1949-1953, 2016.

[12] Purcell R, Parks N, Cody J, Hamilton W. Comparison of Wound Complications and Deep Infections with Direct Anterior and Posterior Approaches in Obese Hip Arthroplasty Patients. J Arthroplasty, (33) 220-223, 2018.

[13] Ponzio D, Poultsides L, Salvatore A, Lee Y, Memtsoudis S, Alexiades M. In-Hospital Morbidity and Postoperative Revisions After Direct Anterior vs Posterior Total Hip Replacement. J Arthroplasty 1421-1425, 2017.

[14] Parvizi J, Restrepo C, Maltenfort MG. Total Hip Arthroplasty Performed Through Direct Anterior Approach Provides Superior Early Outcome. Orthop Clin North Am. 47(3):497-504, 2017.

[15] Moskal JT, Capps SG, Scanelli JA. Anterior muscle sparing approach for total hip arthroplasty. World J Orthop, 4(1):12, 2013.

[16] Berend KR, Lombardi AV, Seng BE, et al. Enhanced early outcomes with the anterior supine intermuscular approach in primary total hip arthroplasty. J Bone Joint Surg Am, 91(Suppl. 6):107, 2009.

[17] Russo MW, Macdonell JR, Paulus MC, Keller JM, Zawadsky MW. Increased Complications in Obese Patients Undergoing Direct Anterior Total Hip Arthroplasty. J Arthroplasty. (30): 1384-1387, 2015.

[18] Sculco TP, Boettner F. Minimally invasive total hip arthroplasty: the posterior approach. Instr Course Lect. 55:205-214., 2006.

[19] Bozic KJ, Ward DT, Lau EC, et al. Risk factors for periprosthetic joint infection following primary total hip arthroplasty: a case control study. J Arthroplasty. 29(1):154-156, 2014.

[20] Elson LC, Barr CJ, Chandran SE, Hansen VJ, Malchau H, Kwon Y-M. Are morbidly obese patients undergoing total hip arthroplasty at an increased risk for component malpositioning? J Arthroplasty. 28(8 Suppl):41-44, 2013.

[21] Berend KR, Mirza AJ, Morris MJ, Lombardi AV Jr. Risk of Periprosthetic Fractures with Direct Anterior Primary Total Hip Arthroplasty. J Arthroplasty. 31(10):2295-2298, 2016.

[22] Ponzio DY, Poultsides LA, Salvatore A, Lee Y-Y, Memtsoudis SG, Alexiades MM. In-Hospital Morbidity and Postoperative Revisions After Direct Anterior vs Posterior Total Hip Arthroplasty. J Arthroplasty, 2017.

[23] Restrepo C, Parvizi J, Pour AE, et al. Prospective randomized study of two surgical approaches for total hip arthroplasty. J Arthroplasty, 25:671, 2010.

[24] Nakata K, NishikawaM, Yamamoto K, et al. A clinical comparative study of the direct anterior with mini-posterior approach: two consecutive series. J Arthroplasty, 24:698, 2009.
[25] Oosting E, Hoogeboom TJ, Dronkers JJ, Visser M, Akkermans RP, van Meeteren NLU. The Influence of Muscle Weakness on the Association between Obesity and Inpatient Recovery from Total Hip Arthroplasty. J Arthroplasty. 32(6):1918-1922, 2017.

[26] Hanna SA, McCalden RW, Somerville L, Howard JL, Naudie DD, MacDonald SJ. Morbid Obesity Is a Significant Risk of Failure Following Revision Total Hip Arthroplasty. J Arthroplasty. 32(10):3098-3101, 2017.

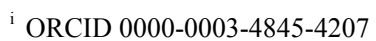

